\title{
L'accès à l'enseignement préprimaire permet-il d'améliorer les résultats scolaires ?
}

\section{POINTS CLÉS}

- Les élèves de 15 ans ayant suivi un enseignement préprimaire obtiennent de meilleurs résultats aux évaluations PISA que les autres, même après contrôle du milieu socio-économique.

- L'accès à l'enseignement préprimaire est moins facile pour les élèves issus de milieux défavorisés que pour les élèves issus de milieux favorisés dans presque tous les pays, notamment ceux où l'enseignement préprimaire est peu répandu.

- Les systèmes d'éducation performants et équitables sont aussi ceux où les inégalités socioéconomiques sont les plus faibles en termes d'accès à l'enseignement préprimaire.

- Les modalités d'accueil de l'enseignement préprimaire influent sur l'ampleur des bénéfices que les élèves peuvent en tirer.

Les bénéfices de l'enseignement préprimaire sont manifestes et quasi universels.
C'est une évidence : I'enseignement préprimaire est bénéfique pour les élèves. Les résultats de l'enquête PISA 2009 montrent que, dans la quasi-totalité des pays de l'OCDE, les élèves de 15 ans ayant suivi un enseignement préprimaire obtiennent de meilleurs résultats que les autres. Ainsi, l'écart de score entre les élèves ayant suivi un enseignement préprimaire pendant plus d'un an et ceux n'ayant pas du tout suivi ce type d'enseignement s'élève à 54 points sur l'échelle PISA de compréhension de l'écrit - soit plus d'une année d'études (qui équivaut à 39 points). Si la plupart des élèves ayant suivi un enseignement préprimaire sont issus de milieux favorisés, l'écart de performance persiste même lorsque l'on compare des élèves issus de milieux similaires. Après contrôle du milieu socio-économique, les élèves ayant suivi un enseignement préprimaire obtiennent un score supérieur de 33 points, en moyenne, à celui des élèves n'ayant pas suivi ce type d'enseignement.

En Belgique, en France et en Israël, les élèves qui ont déclaré avoir suivi un enseignement préprimaire pendant plus d'un an ont obtenu un score supérieur de 100 points au moins sur l'échelle PISA de compréhension de l'écrit à celui des autres élèves. Si l'on compare des élèves issus de milieux similaires, cet écart se réduit mais reste toutefois supérieur à 60 points. En revanche, dans d'autres pays comme l'Estonie, la Finlande, la Corée et les États-Unis, la fréquentation d'un établissement préprimaire ne présente qu'une faible relation - voire aucune - avec les performances ultérieures des élèves issus d'un même milieu socio-économique. 


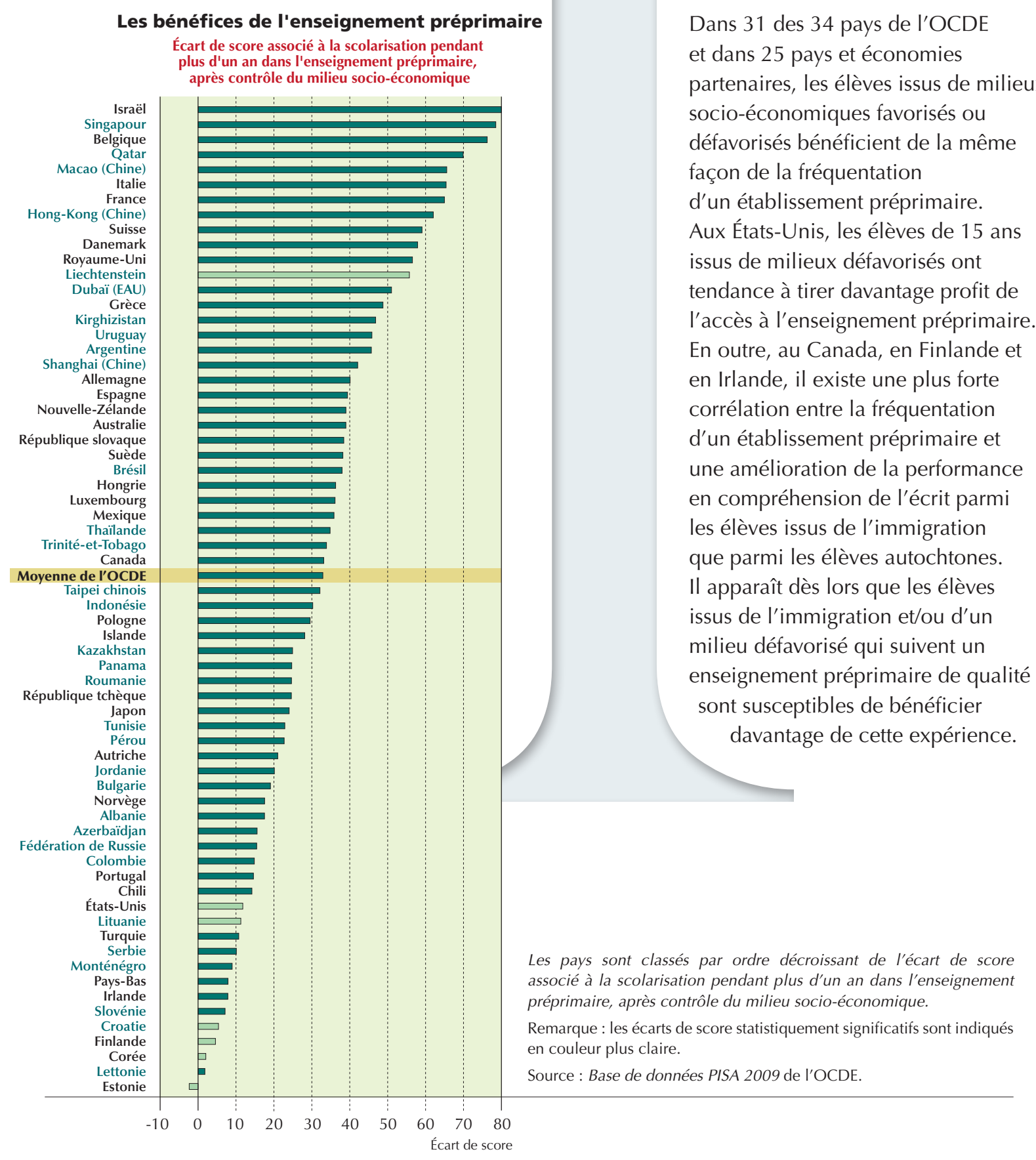

Dans 31 des 34 pays de l'OCDE et dans 25 pays et économies partenaires, les élèves issus de milieux favorisés ou même Aux États-Unis, les élèves de 15 ans ssus de milieux défavorisés ont l'accès à l'enseignement preprimaire. En outre, au Canada, en Finlande et en Irlande, il existe une plus forte corrélation entre la fréquentation d'un établissement préprimaire et une amélioration de la performance élèves issus de l'immigration que parmi les élèves autochtones. Il apparaît dès lors que les élèves issus de l'immigration et/ou d'un milieu défavorisé qui suivent un enseignement préprimaire de qualité sont susceptibles de bénéficier davantage de cette expérience.

\section{L'accès à l'enseignement}

préprimaire est répandu dans

de nombreux pays de l'OCDE...

Les résultats du PISA 2009 montrent qu'en moyenne, dans les pays de l'OCDE, $72 \%$ des élèves de 15 ans évalués dans le cadre de l'enquête PISA déclarent avoir suivi plus d'une année d'enseignement préprimaire. En Belgique, en France, en Hongrie, en Islande, au Japon et aux Pays-Bas, I'enseignement préprimaire est quasi universel, avec plus de $90 \%$ des élèves déclarant avoir fréquenté un établissement préprimaire pendant plus d'un an. Par ailleurs, plus de 90 \% des élèves dans 27 pays de l'OCDE affirment avoir suivi un enseignement préprimaire au moins pendant quelque temps. 
Toutefois, I'enseignement préprimaire reste peu répandu en Turquie, où moins de $30 \%$ des élèves de 15 ans déclarent avoir fréquenté un établissement préprimaire pendant au moins un an. De même, au Canada, au Chili, en Irlande et en Pologne, moins de $50 \%$ des élèves ont suivi un enseignement préprimaire pendant plus d'un an.

\section{Enseignement préprimaire}

Par enseignement préprimaire, on entend toutes les formes d'activités organisées et régulières se déroulant dans des structures adéquates - telles que les écoles maternelles, crèches et garderies et visant à renforcer le développement cognitif, émotionnel et social des enfants. En général, ces programmes sont accessibles aux enfants à partir de trois ans.
... mais dans les pays de l'OCDE, les élèves qui ont suivi un enseignement

préprimaire sont souvent issus de

milieux plus favorisés que ceux qui n'ont pas suivi ce type d'enseignement.

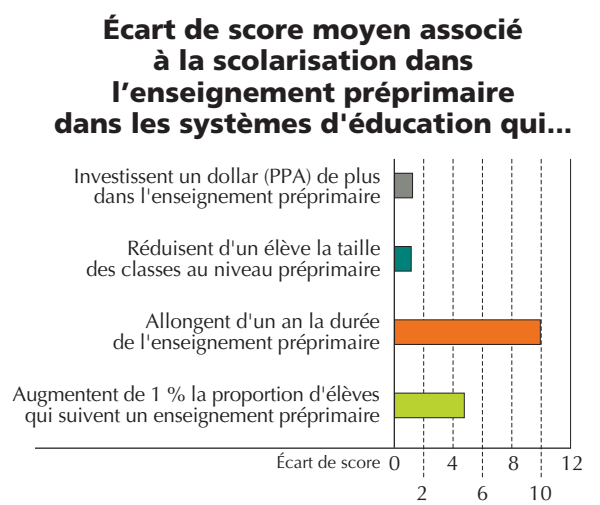

Parmi les raisons à l'origine de ce déséquilibre, citons les coûts nets élevés de la garde d'enfants dans certains pays (en Irlande par exemple), la capacité d'accueil limitée dans les structures de garde d'enfants subventionnées (au Portugal), ou encore le fait que les enfants issus de l'immigration sont, à cet âge, moins susceptibles d'utiliser les modes de garde formels, à moins que leur fréquentation ne soit obligatoire (en Belgique et aux Pays-Bas).

Les résultats du PISA suggèrent que les systèmes d'éducation les plus performants et les plus équitables en termes d'égalité des chances dans l'apprentissage sont aussi ceux qui offrent l'accès le plus large à I'enseignement préprimaire. Par exemple, le Japon, la Corée, l'Estonie, I'Islande et Hong-Kong (Chine) disposent de systèmes d'éducation dans lesquels l'écart de milieu socio-économique entre les élèves ayant suivi un enseignement préprimaire et les autres est inférieur à la moyenne. Parmi les pays dont les performances et l'équité sont en-deçà de la moyenne, seule la Bulgarie fait état d'un accès à l'enseignement préprimaire plus inclusif que la moyenne.

Les modalités d'accueil de l'enseignement préprimaire influent sur

le degré de corrélation entre la fréquentation d'un établissement

préprimaire et les résultats d'éducation à l'ầge de 15 ans.

L'enquête PISA 2009 montre que la relation entre la fréquentation d'un établissement préprimaire et I'amélioration de la performance des élèves à l'âge de 15 ans est la plus forte dans les systèmes d'éducation où ce type d'enseignement est proposé à une plus large proportion d'élèves, pendant une plus longue période de temps, avec des taux d'encadrement supérieurs et un investissement par élève plus important à ce niveau d'enseignement.

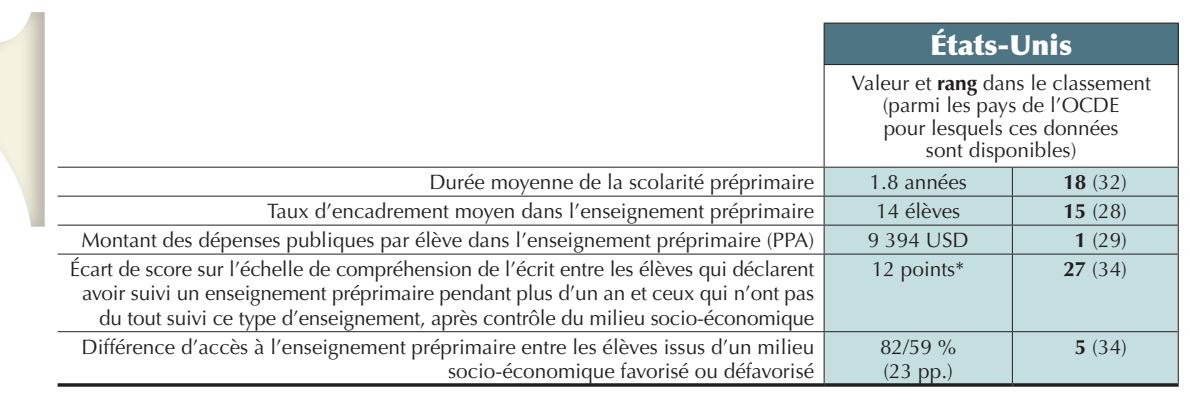

*Écart non statistiquement significatif 


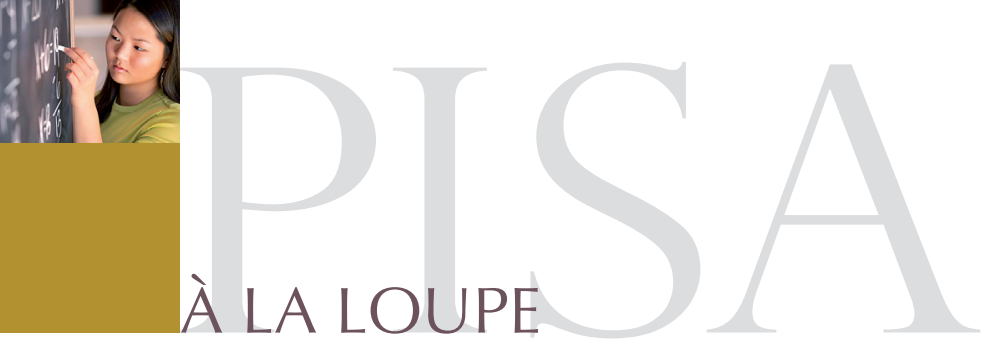

Les bénéfices tirés de l'enseignement préprimaire entraînent-ils une amélioration des performances dans les systèmes d'éducation où davantage d'élèves ont accès à ce niveau d'enseignement ? Bien que les résultats du PISA ne montrent pas de lien entre la performance moyenne des pays de l'OCDE et la proportion d'élèves de ces pays qui ont suivi un enseignement préprimaire, si l'on considère tous les pays et économies ayant participé au PISA 2009, on observe une relation positive entre cette même proportion et la performance du pays concerné, et ce même après contrôle du PIB. À titre d'exemple, les systèmes d'éducation qui affichent un avantage de 10 points de pourcentage dans la proportion d'élèves ayant suivi un enseignement préprimaire obtiennent un score supérieur de 12 points, en moyenne, sur l'échelle PISA de compréhension de l'écrit.

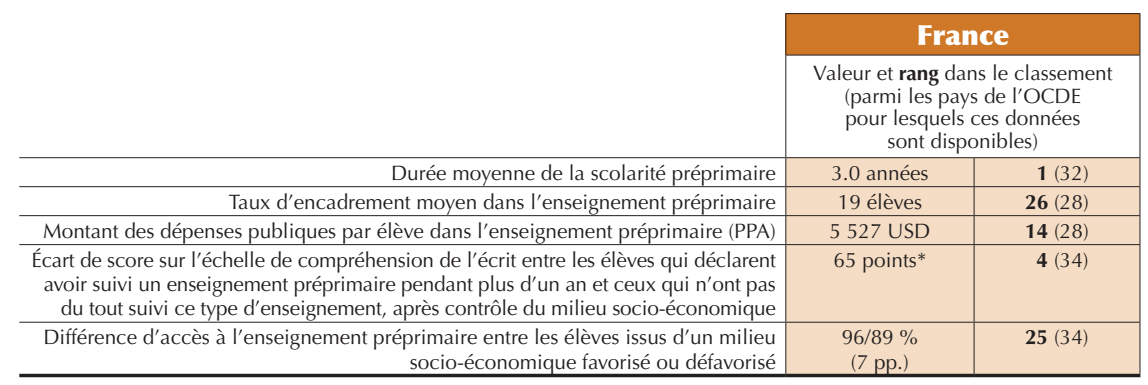

De plus en plus de chercheurs s'accordent à dire que l'éducation de la petite enfance permet d'améliorer le bien-être des élèves, de poser les fondations de l'apprentissage tout au long de la vie, d'accroître l'égalité des chances dans l'éducation, de réduire la pauvreté et de faire progresser la mobilité sociale d'une génération à I'autre. Les résultats du PISA suggèrent qu'il existe une corrélation particulièrement forte entre la participation à l'enseignement préprimaire et les performances en compréhension de l'écrit à l'âge de 15 ans dans les pays qui ont mis en place des politiques pour améliorer la qualité de ce niveau d'enseignement. Forte de ces constatations, l'OCDE élabore actuellement des outils d'action publique en ligne afin d'aider les décideurs à définir et à mettre en œuvre des politiques permettant d'améliorer la qualité de l'éducation et des soins à la petite enfance.

Pour conclure : élargir l'accès à l'enseignement préprimaire permet d'améliorer à la fois la performance globale et l'équité en réduisant les disparités socioéconomiques entre élèves, à condition que cet élargissement ne se fasse pas au détriment de la qualité de l'enseignement.

\section{Pour tout complément d'information}

Contacter Miyako Ikeda (Miyako.Ikeda@oecd.org) ou Pablo Zoido (Pablo.Zoido@oecd.org).

Consulter Résultats du PISA 2009, Surmonter le milieu social : L'égalité des chances dans l'apprentissage et l'équité du rendement de l'apprentissage (volume II) et Résultats du PISA 2009, Les clés de la réussite des établissements d'enseignement : Ressources, politiques et pratiques (volume IV).

\section{Voir}

www.pisa.oecd.org

www.oecd.org/edu/earlychildhood

www.oecd.org/edu/earlychilhood/quality

www.oecd.org/els/social/family/database
Prochain numéro

Améliorer la performance:

Le succès viendra d'en bas 